\title{
Estudio Cinético del Efecto de Polifenilsulfona Sobre el Curado de una Resina Epoxi/Amina Mediante Calorimetría Diferencial de Barrido Convencional y Modulada con Temperatura. Parte II
}

Asdrúbal J. Cedeño

Universidad de Oriente, Venezuela

\author{
Humberto Vázquez-Torres \\ Universidad Autónoma Metropolitana, México
}

\begin{abstract}
Resumen: En este trabajo se estudió el efecto de la adición del termoplástico lineal polifenilsulfona (PPSU), sobre la cinética de reacción y las propiedades térmicas de una resina epoxídica basada en diglicidil éter de bisfenol - A (DGEBA), curada con diaminodifenilsulfona (DDS). El estudio cinético y la caracterización se realizaron mediante calorimetría diferencial de barrido, DSC estándar y modulado, bajo condiciones isotérmicas y dinámicas. La cinética del curado se discutió en el marco de tres modelos cinéticos: Kissinger, Flynn-Wall-Ozawa y el modelo cinético de orden $n$. Para describir la reacción de curado en su última etapa, se usó la relación semiempírica propuesta por Chern y Poehlein para considerar la influencia de la difusión sobre la rapidez de reacción. El mecanismo de curado, para todos los sistemas, se ajustó a una cinética de orden $n$, a pesar del contenido de PPSU, y se observó que éste se hace muy controlado por la difusión conforme aumenta el contenido de PPSU y conforme la temperatura de curado disminuye. El tiempo de vitrificación de los sistemas exhibió una fuerte dependencia con el contenido de PPSU.
\end{abstract}

Palabras-claves: Cinética, difusión, reacción.

\section{Kinetic Study on the Effect of Curing Polyphenylsulfone Epoxy Resin / Amina by Differential Calorimetry Scanning Conventional and Modulated Temperature. Part II}

\begin{abstract}
In this work we studied the effect of the addition of the linear thermoplastic polyphenyl sulfone (PPSU) on the cure kinetics and the thermal properties of a resin based on diglycidyl ether of bisphenol-A (DGEBA), cured with 4,4'-diaminodiphenyl sulfone (DDS). The kinetic study and the characterization process have been carried out by using differential scanning calorimetry, DSC, and temperature modulated DSC (TMDSC), under isothermal and dynamic conditions. The curing kinetics was discussed in the framework of three kinetic models: Kissinger, Flynn-Wall-Ozawa, and the model of reaction of order $n$. To describe the cured reaction in its last stage, we have used the semiempirical relationship proposed by Chern and Poehlein to take into account the influence of diffusion on the reaction rate. The cure mechanism for the studied system obeyed an order $n$ reaction kinetics, regardless of PPSU content, and all of them become far more diffusion controlled at higher contents of PPSU and lower cure temperatures. The vitrification time of the resins exhibited a strong dependence on the PPSU content in the semi-IPN systems.
\end{abstract}

Keywords: Kinetic, diffusion, reaction.

\section{Introducción}

En muchos casos, el proceso de curado de materiales termofijos involucra la conversión de líquidos, de bajo peso molecular, a polímeros amorfos de alto peso molecular o redes tridimensionales conforme avanza la reacción química exotérmica ${ }^{[1]}$. El estado del curado de un polímero termofijo se refiere a lo extenso que ha avanzado el entrecruzamiento a lo largo de la red polimérica. La densidad de entrecruzamiento y la estequiométrica extensión de la reacción caracterizan el estado del curado y consecuentemente las propiedades mecánicas de la red resultante. En última instancia, el estado del material es gobernado por la conversión química y la temperatura de curado. La gelación y la vitrificación, dos fenómenos distintos, juegan un papel muy importante en el procesamiento y el curado de los materiales termofijos. En este punto, las limitaciones producidas por la difusión controlan las reacciones posteriores, debido a la obstaculización de la movilidad molecular, y el sistema se hace rígido conduciendo a una conversión final menor que la unidad. Por otro lado, las resinas epoxídicas son importantes como matrices rígidas para el moldeado de compuestos. Además, las resinas epoxídicas termofjas son conocidas, generalmente, como materiales frágiles y han sido endurecidas añadiéndoles hules reactivos o termoplásticos dúctiles de alto rendimiento antes del proceso de curado. Lo último aparece como una valiosa estrategia para mejorar su dureza sin sacrificar otras propiedades útiles tales como la temperatura de transición vítrea y la rigidez ${ }^{[2-14]}$. La mayoría de los estudios en sistemas epoxi/amina modificados con termoplásticos han sido focalizados en sus propiedades mecánicas y sus morfologías finales ${ }^{[5]}$ y sólo unos pocos de ellos, recientemente, han investigado el efecto del termoplástico lineal sobre la cinética del curado $^{[10,15-18]}$. Actualmente, muchos investigadores profundizan en el estudio del proceso del curado, así como de las relaciones estructura - propiedades de las resinas epoxídicas curadas, con la finalidad de obtener un mejor control del curado y optimizar las propiedades físicas de los productos finales ${ }^{[2]}$. Este artículo es el segundo de una serie, en la cual se

Autor para correspondência: Asdrúbal J. Cedeño, Departamento de Física, Laboratorio de Polímeros, Núcleo de Sucre, Universidad de Oriente, CP 6101, Cumaná, Sucre, Venezuela. E-mail: asjece@gmail.com 
reportará el estudio de la cinética de curado y las propiedades mecánicas finales de diferentes sistemas epoxi/amina modificados con polifenilsulfona. El primero se focalizó en detallar la cinética del curado de sistemas epoxídicos basados en diglicidil éter de bisfenol-A (DGEBA) curados con 4,4'-diaminodifenilmetano $(\mathrm{DDM})^{[19]}$. En este trabajo, la técnica de DSC, tanto convencional como la modulada por temperatura (TMDSC), fue usada para estudiar la cinética de curado de un sistema epoxídico basado en diglicidil éter de bisfenol-A (DGEBA) curada con 4,4'-diaminodifenilsulfona (DDS). Este sistema y sus mezclas con el termoplástico lineal polifenilsulfona (PPSU) a 0, 5, 10 y $15 \mathrm{phr}$ (donde phr representa el número de partes por ciento de la resina DGEBA) fueron además estudiados. Los resultados se analizaron mediante la aplicación de dos modelos dinámicos, el modelo de Kissinger ${ }^{[20]}$ y el modelo de Flynn-Wall-Ozawa ${ }^{[21]}$, y del modelo de reacción de orden $n$.

\section{Desarrollo Experimental}

\section{Materiales}

Los materiales usados en este trabajo fueron el prepolímero epoxídico diglicidil éter de bisfenol-A (DGEBA), curado con diaminodifenilsulfona (DDS). El termoplástico lineal fue polifenilsulfona (PPSU), el cual es un termoplástico amorfo de alto desempeño que ofrece mejor resistencia al impacto y superior resistencia química que muchos otros termoplásticos amorfos de alto rendimiento. Todos los materiales fueron adquiridos de Aldrich Chemical Co. (St. Louis, MO, U.S.A). En la Tabla 1 se dan algunas características relevantes de estos materiales.

\section{Preparación de las muestras}

Para la preparación de las muestras de termofijos (sistema epoxi/ amina) y de semi-IPN se usó una proporción estequiométrica 1:1, para ello se determinó el equivalente químico, en peso, tanto del compuesto epoxídico como del agente de curado. Así, se pesaron cantidades de los prepolímeros y se mezclaron de la siguiente manera:

1. Preparación de la resina pura: La resina epoxídica DGEBA, se calentó a $135^{\circ} \mathrm{C}$ (temperatura mínima necesaria para lograr la mezcla con la DDS) y luego se añadió, el respectivo agente endurecedor (DDS), con agitación mecánica continua durante 5 minutos, el cual fue suficiente tiempo para obtener una mezcla homogénea.

2. Preparación de sistemas semi-IPN: Diferentes cantidades pesadas de PPSU, necesarias para obtener 5, 10 y $15 \mathrm{phr}$ de PPSU en las respectivas mezclas, se disolvieron en $50 \mathrm{~mL}$ de diclorometano a temperatura ambiente; donde phr significa partes por ciento de la resina DGEBA. El material epoxídico, DGEBA, fue entonces añadido lentamente y agitado mecánicamente y de manera continua hasta obtener la completa disolución de la resina. Después, el solvente fue removido calentando a $80{ }^{\circ} \mathrm{C}$ por 24 horas en vacío. Para agregar la requerida cantidad estequiométrica del agente de curado la mezcla DGEBA/PPSU se calienta a $135^{\circ} \mathrm{C}$ y la DDS, previamente calentada a la misma temperatura, se añade lentamente y bajo agitación continua durante 5 minutos.

\section{Mediciones calorimétricas}

Las mediciones calorimétricas, convencional y modulada (referidas como TMDSC), se realizaron usando un calorímetro diferencial de barrido DSC 2920 fabricado por TA Instruments (New Castle, DE, USA). Los experimentos isotérmico y no-isotérmicos, se realizaron con muestras de aproximadamente $10 \mathrm{mg}$ bajo un flujo de nitrógeno de $50 \mathrm{~mL} \cdot \mathrm{min}^{-1}$. Para las mediciones dinámicas se realizaron corridas a diferentes velocidades de calentamiento $\left(2.5,5,10,15\right.$ y $\left.20{ }^{\circ} \mathrm{C} / \mathrm{min}\right)$ en el intervalo de temperatura de 30 a $350^{\circ} \mathrm{C}$. Para las mediciones isotérmicas moduladas se realizaron corridas a diferentes temperaturas $\left(140,160,180\right.$ y $\left.200{ }^{\circ} \mathrm{C}\right)$ con una amplitud de temperatura de $1{ }^{\circ} \mathrm{C}$ y un periodo de 60 segundos.

\section{Técnicas de análisis para los datos DSC}

Los datos obtenidos mediante las mediciones DSC se analizaron usando las dos presentaciones de ésta técnica: la isotérmica y la dinámica.

\section{Técnica isotérmica}

Una manera directa para medir el grado de curado, o extensión de la conversión, $\alpha$, en función del tiempo durante una reacción química, es monitoreando el cambio de calor, o entalpía, durante la reacción en función del tiempo a una temperatura constante a la cual la muestra reacciona dentro de la celda prevista en un equipo DSC. El grado de curado, o simplemente, la conversión, de una resina termofija se relaciona con el calor de reacción mediante la Ecuación 1:

$$
\alpha=\frac{\Delta H(t)}{\Delta H_{0}}
$$

donde $\Delta H(t)$ es la entalpía a un cierto tiempo en un experimento isotérmico y $\Delta H_{0}$ es la entalpía total, obtenida de experimentos dinámicos. Además, durante el proceso de curado se supone que el calor generado es proporcional al consumo del equivalente epoxídico a un tiempo dado.

\section{Técnica de barrido dinámico de temperatura}

La técnica de barrido dinámico de temperatura, o DSC dinámico, monitorea el flujo de calor conforme la temperatura varía linealmente con el tiempo. Usualmente, el uso de esta técnica involucra la suposición de un calor específico constante o una variación lineal del calor específico con un barrido de temperatura. En el barrido dinámico de temperatura, tanto la temperatura de curado como la conversión varían simultáneamente.

\section{Resultados y Discusión}

En la Figura 1 se muestran los gráficos del calor de reacción en función del tiempo para los diferentes sistemas DGEBA/DDS/PPSU con $0,5,10$ y 15 phr del termoplástico lineal PPSU, durante el curado isotérmico a diferentes temperaturas de curado. Como puede verse, la entalpía total disminuye ligeramente con el contenido de PPSU para las temperaturas de curado de 160 y $180{ }^{\circ} \mathrm{C}$, y disminuye más notoriamente cuando el curado se efectúa a $200{ }^{\circ} \mathrm{C}$. Es importante destacar el hecho de que el tiempo de curado prácticamente no fue sensible al contenido del termoplástico lineal. Este comportamiento indica que el PPSU no reacciona en estos sistemas, tal como se

Tabla 1. Algunas características, suministrada por Aldrich Chemical Co., de los materiales usados.

\begin{tabular}{|c|c|c|c|c|c|c|}
\hline Componentes & $M_{n}\left(\right.$ g.mol $\left.{ }^{-1}\right)$ & $\mathbf{T}_{\mathrm{g}}\left({ }^{\circ} \mathrm{C}\right)$ & Densidad (g.cm-3) & $\mathbf{T}_{\mathrm{m}}\left({ }^{\circ} \mathbf{C}\right)$ & Funcionalidad & Equiv $\left(\mathrm{g}^{-1}\right)$ \\
\hline DGEBA & 374 & - & 1.170 & - & 2 & 187.0 \\
\hline DDS & 248.31 & - & - & $175-179$ & 4 & 62.1 \\
\hline PPSU & $21-23 \times 10^{3}$ & 208 & 1.290 & - & 0 & 0 \\
\hline
\end{tabular}


esperaba, y sólo afecta ligeramente la completa conversión de los reactantes en las respectivas resinas epoxídicas. También se puede observar que el incremento en la temperatura de curado hace que la rapidez de reacción de los reactantes aumente notoriamente, pero el tiempo de curado aparece invariante. Esto posiblemente se deba al fuerte incremento de la viscosidad, lo cual dificulta las reacciones químicas en el proceso de curado.

Como es sabido, cuando el pico máximo de la isoterma es cercano a $t=0$, es decir, a una conversión $\alpha \sim 0$, el sistema obedece una cinética de orden $n$. Así, de la Figura 1, se puede observar que la máxima evolución de la entalpía de reacción, o reacción de rapidez máxima $(d \alpha / d t)_{\max }$, ocurre al comienzo de la reacción, es decir para $t=0$, lo que corresponde a una conversión aproximadamente igual a cero, $\alpha \sim 0$. Por lo tanto, este sistema obedece a una cinética, o mecanismo, de curado de orden $n$. Costa et al. ${ }^{[22]}$ reportaron que, efectivamente, el sistema DGEBA/DDS sigue un mecanismo de reacción de orden $n$.

Suponiendo que el calor desprendido durante la reacción de polimerización está directamente relacionado con la desaparición de los grupos epoxídicos durante el curado, el área debajo de las exotermas a diferentes tiempos de reacción, que corresponde a la entalpía de reacción evaluada a diferentes valores de tiempo, $\Delta H(t)$, se usó para calcular la conversión fraccionaria, $\alpha(t)$, de la resina epoxídica usando la Ecuación 1. Para determinar la entalpía total de la reacción, $\Delta H_{0}$, las resinas puras DGEBA/DDS y los sistemas DGEBA/DDS/PPSU fueron calentados desde 30 hasta $350{ }^{\circ} \mathrm{C}$ a diferentes rapideces de calentamiento $\left(2.5,5,10,15\right.$ y $\left.20^{\circ} \mathrm{C} / \mathrm{min}\right)$. La Figura 2 exhibe la dependencia de la entalpía con la temperatura para las muestras estudiadas, medidos mediante DSC convencional. La entalpía total de reacción, $\Delta H_{0}$, se determina mediante la integración del área entre el máximo del termograma y la línea recta que conecta la curva antes y después del máximo. De estas mediciones se obtuvieron los valores de $\Delta H_{0}$, necesarios para la determinación del grado de curado. De estas mediciones se calculó

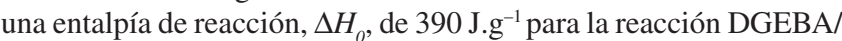
DDS. Este valor es equivalente a $101 \mathrm{~kJ} \mathrm{~mol}^{-1}$ de grupos epoxídicos, en concordancia con los valores hallados en la literatura ${ }^{[9,19,23-25]}$.

Usando la Ecuación 1 y los datos mostrados en las Figuras 1 y 2 , se calculó la conversión, $\alpha(t)$, para todos los sistemas estudiados. La Figura 3 muestra los gráficos del grado de conversión contra el tiempo para una temperatura de curado de $140{ }^{\circ} \mathrm{C}$. Puede observarse claramente que la conversión o grado de curado aumenta rápidamente en las primeras etapas del curado hasta aproximarse asintóticamente a un valor máximo, $\alpha_{\max }$. También puede verse que ninguna de las mezclas alcanza a reaccionar completamente (generalmente, la conversión máxima resultó menor que la unidad) para las temperaturas de curado usadas en esta investigación, y esto es causado por la vitrificación que sufre el sistema en altos valores de conversión.

Una ventaja de la técnica DSC modulada con la temperatura (TMDSC) es que la señal de la capacidad calorífica se puede separar de la señal del flujo de calor. La Figura 4 muestra la evolución del módulo de la capacidad calorífica compleja, $\left|C_{P}^{*}\right|$, para el sistema DGEBA/DDS/PPSU con un contenido de $10 \mathrm{phr}$ del termoplástico lineal a las temperaturas de curado seleccionadas.

Como en otros sistemas de reacción de epoxi/aminas ${ }^{[9,19,26-31]}$, el $\left|C_{P}^{*}\right|$ aumenta inicialmente, permanece constante y después disminuye y se nivela. El pequeño aumento inicial en $\left|C_{P}^{*}\right|$ está asociado a un incremento en el número de estados configuracionales o un incremento de los modos vibracionales durante el crecimiento de las cadenas lineales, mientras que la zona nivelada corresponde al proceso de curado en el cual la red polimérica crece. Finalmente, conforme los grupos aminas secundarios reaccionan, conllevando a

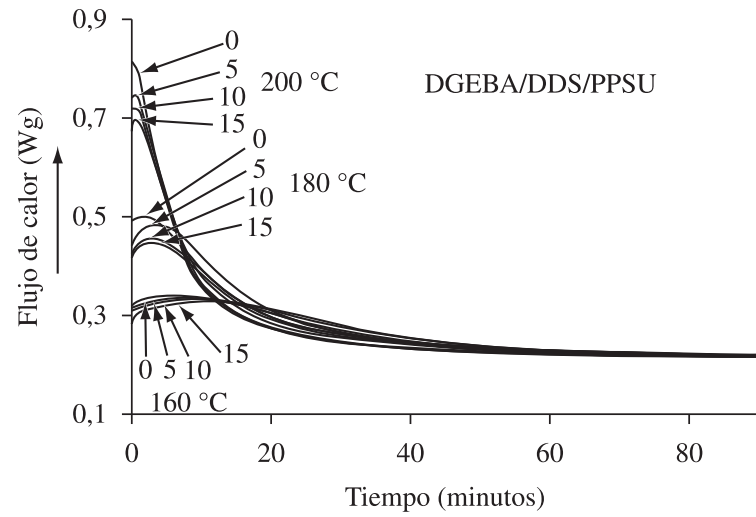

Figura 1. Dependencia del flujo de calor con el tiempo del curado isotérmico, medido con TMDSC, para la resina pura y para los sistemas semi-IPN con 5, 10 y $15 \mathrm{phr}$ de PPSU a las temperaturas de curado de 10,180 y $200^{\circ} \mathrm{C}$.

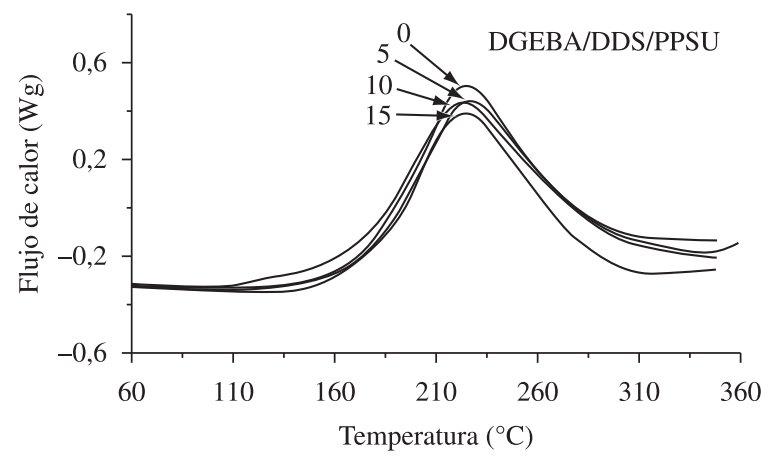

Figura 2. Termogramas de DSC dinámicas para los sistemas estudiados, obtenidos mediante DSC convencional a una rapidez de calentamiento de $10{ }^{\circ} \mathrm{C} / \mathrm{min}$. Se indican las diferentes cantidades (phr) de PPSU.

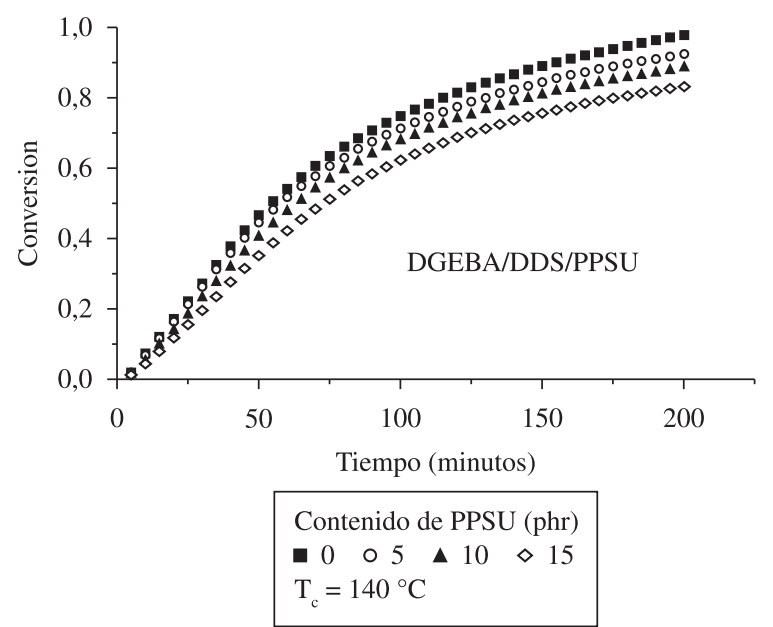

Figura 3. Gráficos de conversión de curado, $\alpha$, contra el tiempo para las muestras durante el curado a una temperatura de $140{ }^{\circ} \mathrm{C} \mathrm{a} 0,5,10$ y $15 \mathrm{phr}$ de PPSU.

la formación de una estructura entrecruzada, ocurre una disminución en el número de estados configuracionales, lo cual induce una disminución en $\left|\mathrm{C}_{P}^{*}\right|$, esto es atribuido a los cambios irreversibles que ocurren cuando el material pasa del estado líquido a un sólido vítreo, lo cual está relacionado con la vitrificación del sistema durante el curado ${ }^{[27-33]}$. El punto de inflexión de las curvas de la Figura 4 corresponde a un tiempo de relajación característico, llamado tiempo de vitrificación, $t_{v}$, donde el número de estados configuracionales 
disminuye considerablemente y surge una abrupta disminución en la capacidad calorífica del sistema. En este tiempo el sistema logra una temperatura de transición vítrea, $T_{g}$, superior a la temperatura de polimerización que se aplica al sistema en estudio. De estas curvas también se pueden determinar el comienzo y el final del proceso de vitrificación, obteniendo así el intervalo de vitrificación.

En la Tabla 2 se dan los tiempos de vitrificación, determinados como los puntos de inflexión de las curvas del módulo de la capacidad calorífica contra el tiempo de curado, para todos los sistemas estudiados y para todas las temperaturas de curado. Se puede observar que los tiempos de vitrificación disminuyen significativamente con el aumento de la temperatura de curado, debido al aumento de la rapidez de reacción, y también disminuyen, de manera más moderada, con el contenido de PPSU. Este último resultado puede atribuirse a una intensificación de la viscosidad del sistema debido a la presencia de las cadenas del termoplástico lineal en la mezcla reactante.

\section{Análisis cinético}

Para el estudio de la cinética del curado se seleccionaron tres modelos para ajustar los datos experimentales y así hallar los parámetros cinéticos. Los modelos cinéticos usados son: 1) dos métodos cinéticos dinámicos: el método de Kissinger y el método de Flynn-Wall-Ozawa, y 2) el modelo de reacción de orden $n$.

\section{Métodos cinéticos dinámicos}

Los dos métodos cinéticos dinámicos se aplicaron a los datos experimentales obtenidos mediante el calentamiento dinámico usando DC convencional. De acuerdo al método de Kissinger, la energía de activación, $E$, se puede obtener del máximo de la rapidez de reacción donde $d(d \alpha / d t) / d t$ es cero para una condición de rapidez de calentamiento constante. De acuerdo a esta consideración, se puede derivar la Ecuación 2:

$$
\frac{d\left[\ln \left(q / T_{m}^{2}\right)\right]}{d\left(1 / T_{m}\right)}=-\frac{E_{a}}{R}
$$

donde $q$ es la rapidez de calentamiento constante y $T_{m}$ es la temperatura correspondiente a la posición del pico exotérmico. Así, la energía de activación se puede obtener de la pendiente de la gráfica de $\ln \left(q / \mathrm{T}_{m}^{2}\right)$ contra $1 / T_{m}$. Por otra parte, Flynn-Wall-Ozawa desarrollaron un método alternativo para determinar la energía de activación y ellos hallaron la Ecuación 3:

$$
\log (q)=\log \left[\frac{A E_{a}}{g(\alpha) R}\right]-2.315-\frac{0.457 E_{a}}{R T_{m}}
$$

donde $\mathrm{g}(\alpha)$ es una función dependiente de la conversión.

De acuerdo con este método, un gráfico de $\log (q)$ contra $1 / T_{m}$ permite obtener la energía de activación sin ninguna suposición acerca de las funciones dependientes de la conversión para diferentes niveles de conversión. La Figura 5 muestra la dependencia con la temperatura para los experimentos de calentamiento dinámicos realizados a las rapideces de calentamiento antes mencionadas para el sistema DGEBA/DDS/PPSU con $10 \mathrm{phr}$ de PPSU.

De estos termogramas de DSC se obtiene la temperatura correspondiente a la posición máxima del pico exotérmico, $T_{m}$, la cual presenta un desplazamiento hacia las temperaturas más altas, mientras que hay una disminución en el calor de polimerización, $\Delta H_{0}$, cuando se incrementa la rapidez de calentamiento. Las energías de activación, obtenidas por los métodos de Kissinger y Flynn-Wall-Ozawa, para los mismos datos de la Figura 5, son listados en la Tabla 3, en la cual puede verse que, como en otros sistemas epoxi/termoplástico reportados ${ }^{[19,34-38]}$, los valores

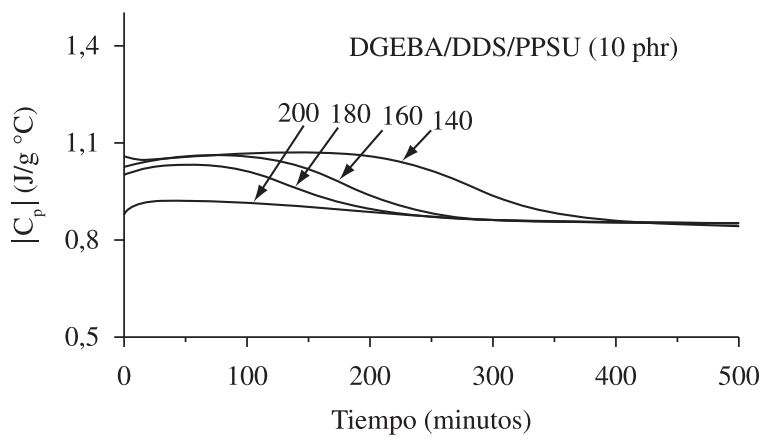

Figura 4. Módulo de la capacidad calorífica compleja correspondiente al curado isotérmico con $10 \mathrm{phr}$ de PPSU, medido con TMDSC y con condiciones de modulación de $1{ }^{\circ} \mathrm{C}$ de amplitud y periodo de 60 segundos. Se indican las temperaturas de curado.

Tabla 2. Tiempos de vitrificación obtenidos para todos los sistemas analizados.

\begin{tabular}{cccccc}
\hline PPSU (phr) & \multicolumn{5}{c}{ Tiempo de vitrificación, $\boldsymbol{t}_{\boldsymbol{v}}$ (minutos) } \\
\cline { 2 - 6 } & $\mathbf{T}_{\text {curado }}\left({ }^{\circ} \mathbf{C}\right)$ & $\mathbf{1 4 0}$ & $\mathbf{1 6 0}$ & $\mathbf{1 8 0}$ & $\mathbf{2 0 0}$ \\
\hline 0 & 298.26 & 184.08 & 125.64 & 115.03 \\
5 & 292.88 & 181.84 & 121.66 & 110.35 \\
10 & 287.45 & 176.44 & 119.82 & 107.05 \\
15 & 280.21 & 174.93 & 115.71 & 105.65 \\
\hline
\end{tabular}

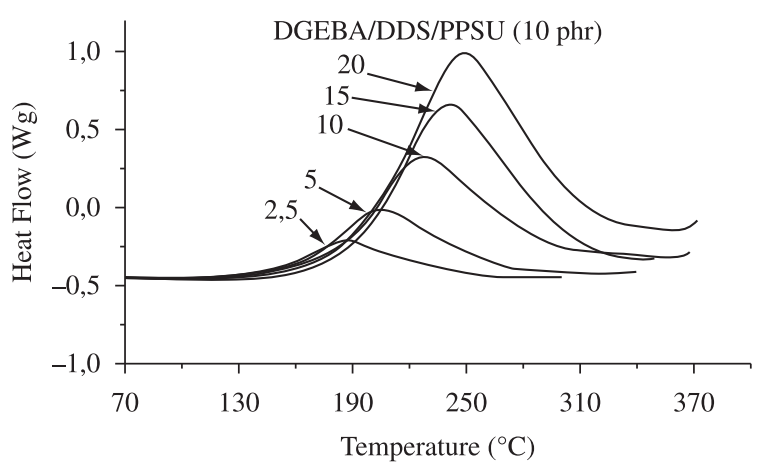

Figura 5. Dependencia con la temperatura del flujo de calor para el semi-IPN con 10 phr de PPSU a las rapideces de calentamiento de 2.5, 5, 10, 15 y $20{ }^{\circ} \mathrm{C} / \mathrm{min}$.

obtenidos por ambos métodos son muy similares, siendo, no obstante, ligeramente más grandes los valores obtenidos por el método de Flynn-Wall-Ozawa que por el método de Kissinger. Los resultados obtenidos permiten concluir que la energía de activación no presenta un marcado cambio con el contenido del termoplástico lineal para todos los sistemas estudiados.

\section{Cinética de curado de orden n}

Para una reacción química de orden $n$ la ecuación de la rapidez de reacción puede escribirse como (Ecuación 4):

$$
\frac{d \alpha}{d t}=k(1-\alpha)^{n}
$$

Es conveniente expresar esta ecuación en forma logarítmica (Ecuación 5):

$$
\ln \left(\frac{d \alpha}{d t}\right)=\ln k+n \ln (1-\alpha)
$$

La conversión en un proceso de curado es proporcional al cambio en la entalpía; por lo tanto, la conversión, $\alpha$, y la rapidez de conversión, $d \alpha / d t$, pueden obtenerse de las mediciones de la 
Tabla 3. Energías de activación para todos los sistemas estudiados usando el método de Kissinger $\left(E_{l}\right)$ y el método de Flynn-Wall-Ozawa $\left(E_{2}\right)$.

\begin{tabular}{ccc}
\hline PPSU $(\mathbf{p h r})$ & $E_{1}\left(\mathbf{k J} \cdot \mathbf{m o l}{ }^{-1}\right)$ & $E_{2}\left(\mathbf{k J} \cdot \mathbf{m o l} \mathbf{l}^{-1}\right)$ \\
\hline 0 & 57.3 & 60.9 \\
5 & 59.9 & 61.7 \\
10 & 62.3 & 64.5 \\
15 & 64.1 & 66.9 \\
\hline
\end{tabular}

variación del flujo de calor mediante la técnica de DSC tanto en el modo dinámico como en el isotérmico. Así, las Ecuaciones 4 y 5 se pueden usar para el análisis de los datos experimentales obtenidos de corridas dinámicas e isotérmicas y así poder estimar los parámetros cinéticos $k, n$ y $E_{a}$. De acuerdo con la Ecuación 5, para los datos isotérmicos, el orden de reacción para un mecanismo de reacción de orden $n$, se puede obtener de la pendiente del gráfico lineal de $\ln (d \alpha / d t)$ contra $\ln (1-\alpha)$ y la constante de rapidez se obtiene del intercepto con el eje vertical. En este punto, es conveniente señalar que la cinética de orden $n$ es ampliamente usada en el análisis de los datos experimentales obtenidos mediante corridas DSC dinámicas y no dinámicas.

Si los materiales semi-IPN siguen una cinética de orden- $n$, entonces $d \alpha / d t$ es proporcional a la fracción de material sin reaccionar, tal como se puede apreciar en la Ecuación 4. Los sistemas que obedecen cinéticas de este tipo tendrían una rapidez de reacción máxima para $t=0$. Por otro lado, el orden de reacción, $n$, además describe la forma de la corrida isotérmica (función potencial) y su valor influye en la rapidez de reacción de la formulación, lo cual a la vez permite la determinación de los ciclos de curado apropiados para el procesamiento del material compuesto.

En la Figura 6 se muestran las curvas de la rapidez de conversión, $d \alpha / d t$, contra el tiempo de curado para los sistemas curados a una temperatura de $140{ }^{\circ} \mathrm{C}$. Estas curvas muestran el comportamiento típico de los sistemas con mecanismo de reacción de orden $n$, donde la rapidez de reacción parte de un valor máximo a un tiempo muy próximo a cero y luego comienza a disminuir. En estas curvas el máximo de reacción se presenta a tiempos de curado más cortos, lo cual es debido a la naturaleza del mecanismo de reacción para este sistema. Como ya hemos mencionado anteriormente, el máximo que presentan las curvas $d \alpha / d t v s$. $t$ (o $d \alpha / d t v s$. $\alpha$ ) son típicas tanto de los sistemas autocatalíticos como de aquéllos que siguen un mecanismo de reacción de orden $n^{[10,39]}$. Este comportamiento no fue modificado por la presencia del termoplástico, y solamente el máximo en la rapidez de conversión disminuye con el contenido de PPSU. Esto resulta de la contribución adicional que hace la PPSU al incremento de la viscosidad del sistema reactante, debido a su peso molecular relativamente alto, en comparación con el peso molecular de los componentes de la red que se está formando; es decir, cuanto más grande sea el contenido de PPSU, más bajo es el valor del grado de reacción a la cual ocurre el punto aparente de gelación de la resina.

La rapidez de conversión, $d \alpha / d t$, graficada como una función de la conversión, $\alpha$, para los mismos datos de la Figura 1, es mostrada en la Figura 7, a la temperatura de curado de $160{ }^{\circ} \mathrm{C}$. Estas figuras confirman las observaciones hechas en relación al comportamiento de orden $n$ que presentan estas reacciones químicas. En las curvas de la Figura 7 se muestra que la rapidez de reacción, $d \alpha / d t$, presenta un máximo aproximadamente a un $25 \%$ de conversión, sugiriendo que en este punto se ha consumido el $25 \%$ del total de los grupos epoxídicos del sistema. Como es de esperar, $d \alpha / d t$ aumenta significativamente con la temperatura de curado, como puede verse cuando se comparan los valores máximos de las rapideces de conversión para los curados a $160{ }^{\circ} \mathrm{C}$ (Figura 7) y a $180{ }^{\circ} \mathrm{C}$ (Figura 8), cuyos valores son 0.018 y 0.039 , respectivamente, para los sistemas que contienen $15 \mathrm{phr}$ de PPSU. Comparando los mismos

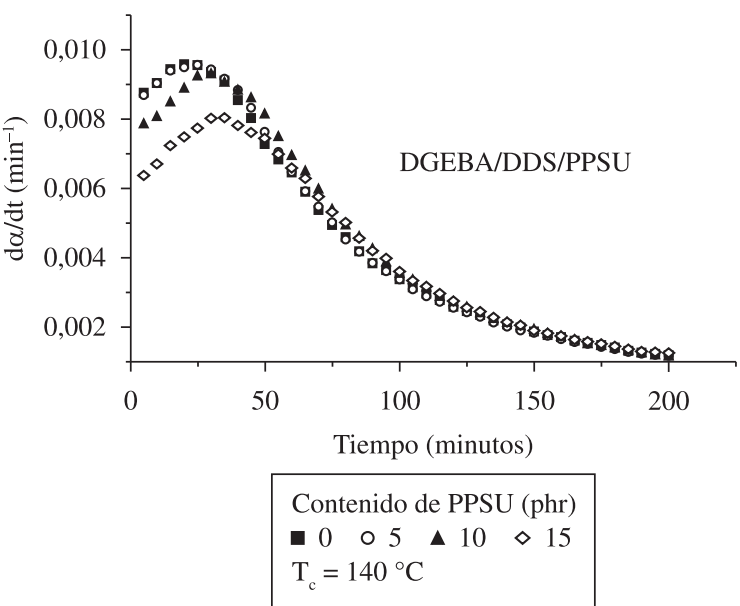

Figura 6. Gráficos de la rapidez de conversión, $d \alpha / d t$, contra el tiempo para los sistemas estudiados durante el curado a una temperatura de $140{ }^{\circ} \mathrm{C}$.

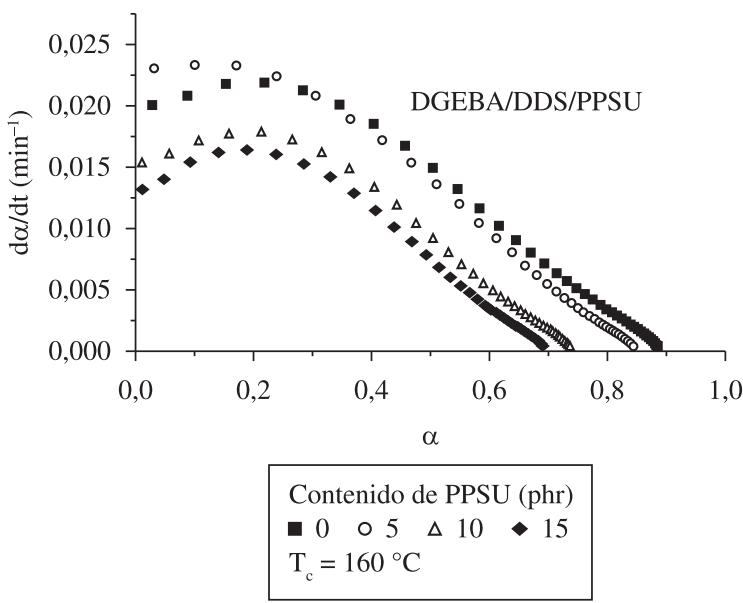

Figura 7. Gráficos de la rapidez de conversión, $d \alpha / d t$, en función de la conversión, $\alpha$, para todos los sistemas estudiados a $160{ }^{\circ} \mathrm{C}$ y con contenidos de PPSU de $0,5,10$ y $15 \mathrm{phr}$.

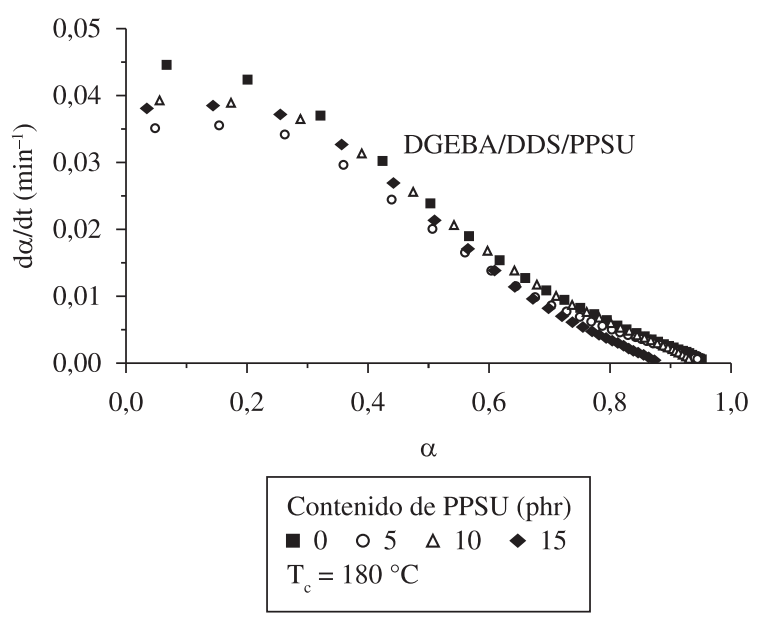

Figura 8. Gráficos de la rapidez de conversión, $d \alpha / d t$, en función de la conversión, $\alpha$, para todos los sistemas estudiados a $160{ }^{\circ} \mathrm{C}$ y con contenidos de PPSU de $0,5,10$ y 15 phr. 
datos para la resina pura en estas figuras, 0.022 (en la Figura 7) y 0.045 (en la Figura 8), se puede ver que la PPSU solo ocasiona un desplazamiento del valor máximo de $d \alpha / d t$ sin otro efecto adicional debido a su inercia química en los sistemas aquí estudiados. De estas curvas se hallan las conversiones máximas, $\alpha_{\max }$, como el intercepto de la curva con el eje de conversión. En la Tabla 4 se presentan los valores hallados para los sistemas a todas las temperaturas de curado isotérmicas usadas. Puede verse que la conversión máxima nunca alcanza la unidad y además disminuye con el contenido del termoplástico en la mezcla. Esto puede atribuirse a la ocurrencia prematura del control por difusión ocasionado por la PPSU.

Los parámetros cinéticos $k$ y $n$, son calculados para cada sistema ajustando los datos experimentales a través de la Ecuación 4 en todo el intervalo de conversión. En todos los sistemas, el ajuste no fue bueno para altas conversiones, $\alpha>0.7$, debido a la vitrificación, la cual hace que el mecanismo de reacción esté controlado por la difusión en lugar de la propia reactividad química de los reactantes ${ }^{[40]}$. Los valores de los parámetros $k$ y $n$, obtenidos de esta manera, son presentados en la Tabla 4. Puede verse de esta tabla que la constante de rapidez, $k$, y el orden de reacción, $n$, aumentan conforme aumenta la temperatura de curado, para todas las mezclas.

Así, considerando estos parámetros cinéticos, los datos experimentales, para todos los sistema estudiados, fueron ajustados al modelo de reacción de orden $n$ y, como puede observarse en la Figura 9, el ajuste fue bueno.

Con la finalidad de considerar los efectos de la vitrificación en el mecanismo de reacción en sistemas epoxi/amina, Chern y Poehlein propusieron una relación semi-empírica, basada en consideraciones de volumen libre, para explicar el control por difusión en las reacciones de curado $^{[41]}$. En esta relación, se define un factor de difusión, $F_{d}(\alpha)$, junto con dos parámetros empíricos, a través de la Ecuación 6:

$$
F_{d}(\alpha)=\frac{1}{1+\exp \left[C\left(\alpha-\alpha_{c}\right)\right]}
$$

donde $C$ es un factor que depende de la temperatura y $\alpha_{c}$ es la conversión crítica. Esto demuestra que, conforme e incrementa la conversión, el efecto de la difusión se desarrolla gradualmente. La rapidez de conversión efectiva a cualquier conversión está definida como la rapidez de conversión química, definida por la Ecuación 4, multiplicada por $F_{d}(\alpha)$ (Ecuación 7):

Tabla 4. Parámetros cinéticos $k$ y $n$, determinados de experimentos isotérmicos mediante el mecanismo de orden $n$.

\begin{tabular}{cccc}
\hline $\mathbf{P h r}$ & $\mathbf{T}_{\mathbf{c}}\left({ }^{\circ} \mathbf{C}\right)$ & $k\left(\mathbf{m i n}^{-1}\right)$ & $\mathbf{n}$ \\
\hline 0 & 140 & 0.011 & 0.67 \\
& 160 & 0.029 & 1.55 \\
& 180 & 0.050 & 1.59 \\
& 200 & 0.120 & 1.71 \\
5 & 140 & 0.012 & 0.93 \\
& 160 & 0.033 & 1.84 \\
& 180 & 0.079 & 2.07 \\
& 200 & 0.115 & 2.53 \\
10 & 140 & 0.012 & 1.08 \\
& 160 & 0.046 & 2.38 \\
& 180 & 0.135 & 2.78 \\
& 200 & 0.157 & 3.06 \\
& 140 & 0.010 & 1.15 \\
& 160 & 0.041 & 2.50 \\
& 180 & 0.086 & 2.87 \\
& 200 & 0.136 & 2.93 \\
\hline
\end{tabular}

$$
\frac{d \alpha}{d t}=k(1-\alpha)^{n} F_{d}(\alpha)
$$

Los datos para $F_{d}(\alpha)$ fueron determinados como la razón de la rapidez de conversión experimental, $(d \alpha / d t)_{\text {exp }}$, y la rapidez de conversión calculada sobre la base del modelo cinético químico, $(d \alpha / d t)_{q u i}$, dada por la Ecuación 4 (Ecuación 8):

$$
F_{d}(\alpha)=\frac{(d \alpha / d t)_{\exp }}{(d \alpha / d t)_{q u i}}
$$

En la Figura 10 se presentan los valores obtenidos para el factor de difusión, $F_{d}(\alpha)$, correspondiente a una temperatura de curado de $160{ }^{\circ} \mathrm{C}$. De estas curvas puede verse que $F_{d}(\alpha)$ tiene un valor aproximado de 1 en las primeras etapas del curado, independientemente del contenido de PPSU, lo cual evidencia que la reacción química está controlada por la reactividad química de los grupos funcionales, es decir, la reacción es químicamente controlada. Conforme el curado progresa, puede observarse una disminución en el factor de difusión, $F_{d}(\alpha)$, y, por lo tanto, en la rapidez de reacción, debido al comienzo del control por difusión. Es importante notar que $F_{d}(\alpha)$ cae a bajas conversiones de curado a medida que aumenta el contenido del termoplástico lineal, PPSU. Los valores de los parámetros $C$ y $\alpha_{c}$ fueron obtenidos mediante el ajuste de los datos experimentales mostrados en la Figura 10, a través de la Ecuación 6, y ellos son presentados en la Tabla 5. Puede verse que el valor de $\alpha_{c}$ aumenta con la temperatura de curado y

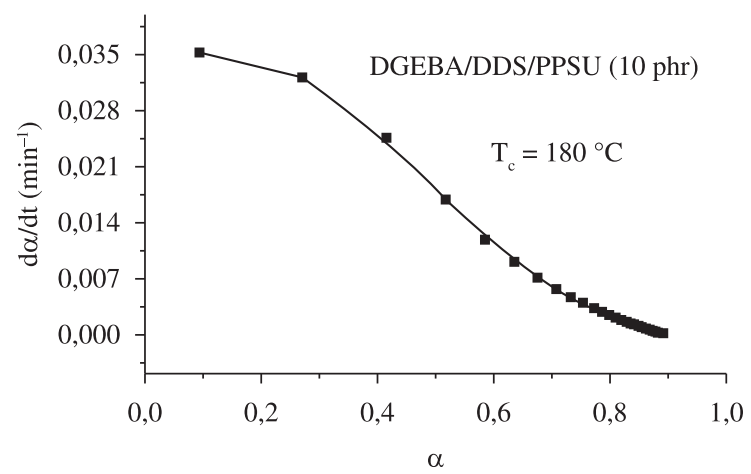

Figura 9. Ajuste de los datos experimentales, mediante el modelo de orden $n$, para la rapidez de conversión isotérmica, $d \alpha / d t$, como una función de la conversión, $\alpha$, curado a $180{ }^{\circ} \mathrm{C}$ y a $10 \mathrm{phr}$ de PPSU. $\mathrm{R}^{2}$ es el factor de correlación del modelo con los datos experimentales.

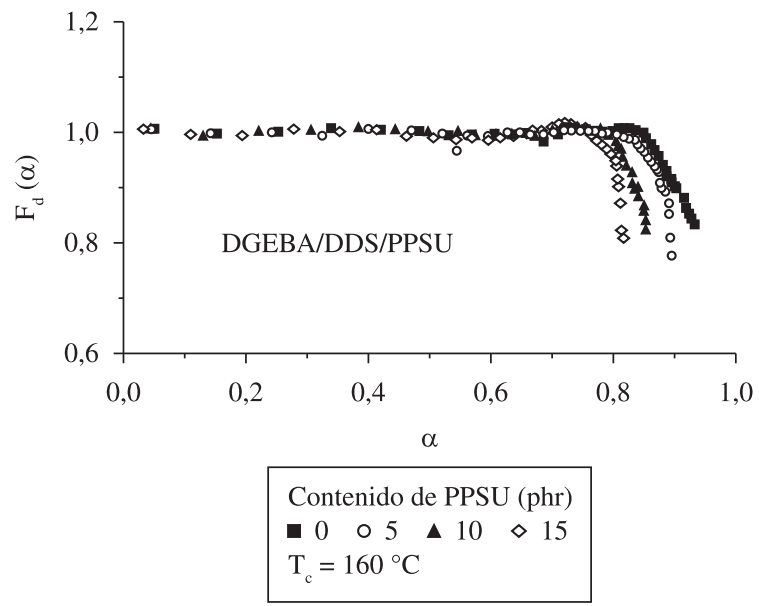

Figura 10. Gráficos del factor de difusión, $F_{d}(\alpha)$, contra la conversión, $\alpha$, para la temperatura de curado de $160^{\circ} \mathrm{C}$. Se indica el contenido de PPSU. 
Tabla 5. Valores de la conversión crítica, $\alpha_{c}$, y del parámetro $C$ para los materiales estudiados.

\begin{tabular}{lccr}
\hline \multicolumn{1}{c}{ Material } & $\mathbf{T}\left({ }^{\circ} \mathbf{C}\right)$ & $\alpha_{c}$ & \multicolumn{1}{c}{$\boldsymbol{C}$} \\
\hline DGEBA/DDS & 140 & 0.93 & 26.40 \\
& 160 & 0.95 & 21.32 \\
& 180 & 0.98 & 85.67 \\
& 200 & 0.99 & 132.77 \\
DGEBA/DDS/PPSU (5 phr) & 140 & 0.89 & 24.93 \\
& 160 & 0.91 & 120.03 \\
& 180 & 0.94 & 121.25 \\
DGEBA/DDS/PPSU (10 phr) & 140 & 0.95 & 217.45 \\
& 160 & 0.81 & 46.86 \\
& 180 & 0.86 & 130.32 \\
& 200 & 0.92 & 147.63 \\
DGEBA/DDS/PPSU (15 phr) & 140 & 0.81 & 394.86 \\
& 160 & 0.83 & 114.49 \\
& 180 & 0.85 & 86.68 \\
& 200 & 0.92 & 104.89 \\
\hline
\end{tabular}

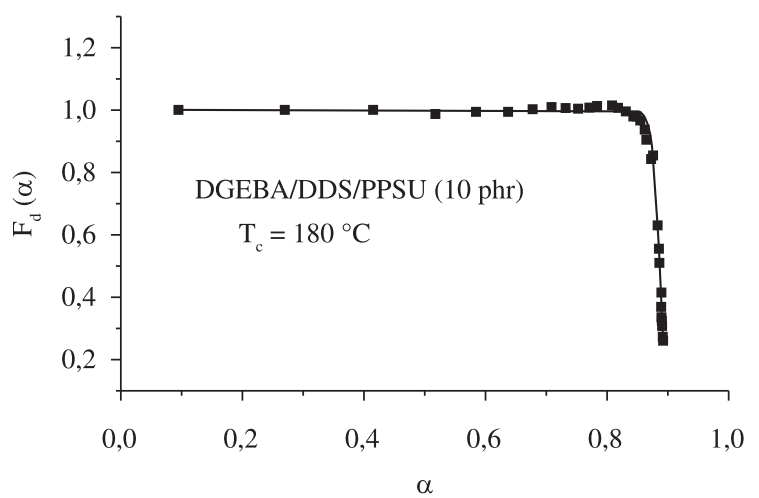

Figura 11. Ajuste de los datos para el factor de difusión, $F_{d}(\alpha)$, para la conversión isotérmica a $180{ }^{\circ} \mathrm{C}$ para el sistema con $10 \mathrm{phr}$ de PPSU. $\mathrm{R}^{2}$ es el factor de correlación del modelo con los datos experimentales.

disminuye con el contenido de PPSU, mientras que para el parámetro $C$ no se halla ninguna tendencia apreciable, en concordancia con los estudios de Cole et al. ${ }^{[42]}$, Barral et al. ${ }^{[10]}$ y Cedeño y Vázquez ${ }^{[19]}$ para sistemas epoxi/amina.

De esta manera, la inclusión del factor de difusión en el modelo de reacción de orden $n$ conduce a una excelente concordancia de este modelo con los resultados experimentales para cada una de las temperaturas seleccionadas. Un ejemplo de este ajuste se muestra en la Figura 11 para la muestra con $10 \mathrm{phr}$ de $\operatorname{PPSU}$, curada a $180{ }^{\circ} \mathrm{C}$.

\section{Conclusiones}

Usando TMDSC y DSC convencional, se ha investigado el proceso de curado isotérmico de un sistema basado en diglicidil éter de bisfenol-A (DGEBA) curado con una diamina basada en 4,4'-diaminodifenilsulfona (DDS) y modificado con polifenil sulfona (PPSU). Se pudo ver que el efecto de la adición de la PPSU sobre la cinética del proceso de curado es muy modesto. A través de modelos simples se notó que la energía requerida por el sistema, en relación a la resina pura, es solo aumentada ligeramente por la presencia del termoplástico lineal. Mediante mediciones con TMDSC, la transición de vitrificación se observó a través del cambio en el módulo de la capacidad calorífica compleja, lo cual es un método directo para estudiar la vitrificación de estos sistemas.
Así, tiempo de vitrificación, a una temperatura seleccionada, se puede obtener de un solo experimento isotérmico. Todos los sistemas estudiados obedecieron a una reacción de curado de orden $n$ en la etapa controlada por la cinética. Para describir el curado en las últimas etapas de la reacción, donde aparece una estructura entrecruzada y la cinética es controlada por la difusión (región postvitrificación), hemos usado una relación semi-empírica propuesta por Chern y Poehlein. Así, el modelo de orden $n$, acoplado con el factor de difusión, puede predecir muy bien todas las etapas de la reacción en todo el intervalo de temperaturas usado. Las conversiones finales disminuyen con el aumento del contenido de PPSU o con la disminución de la temperatura de curado, lo cual conduce a estructuras en redes menos perfectas.

\section{Agradecimientos}

Este trabajo fue financiado por la Universidad Autónoma Metropolitana - Iztapalapa (México D.F, México) y por la Universidad de Oriente (Sucre, Venezuela).

\section{Referencias}

1. Billmeyer, F. - "Textbook of Polymer Science”, John Wiley and Sons (1984).

2. Kim, H. \& Char, K. - Bull Korean Chem. Soc., 20, p.1329 (1999).

3. Bucknall, C. B. \& Gilbert, A. H. - Polymer, 30, p.213 (1989).

4. Venderbosch, R. W.; Meijer, H. \& Lemstra, P. J. - Polymer, 35, p. 4349 (1996).

5. Teng, K. C. \& Chang, F. C. - Polymer, 37, p. 2385 (1996)

6. Tanaka, N.; Lijima, T.; Fukuda, W. \& Tomoi, M. - Polym Int, 42, p.95 (1997).

7. Remiro, P. M.; Riccardi, C. C. \& Corcuera, M. A. - Appl. Polym. Sci., 74, p.772 (1999).

8. Martinez, I.; Martin, M. D.; Eceiza, A. \& Oyanguren, P. - Polymer, 41, p.1027 (2000).

9. Jenninger, W.; Schawe, J. R. \& Alig, I. - Polymer, 41, p.1577 (2000).

10. Barral, L.; Cano, J.; López, J.; López, I. \& Nogueira, P. - Polymer, 41, p.2657 (2000)

11. Varley R. J.; Hodking, J. H.; \& Hawthorne. D. G. - Polymer, 41, p.3425 (2000).

12. Mimura, K.; Ito, H. \& Fujioka, H. - Polymer, 41, p.4451 (2000).

13. Barral, L.; Cano, J. \& López, J. - Polym. Sci., Part B: Polym. Phys., 38, p.351 (2000).

14. Dean, K.; Cook, W. D.; Zipper, M. D. \& Burchill, P. - Polymer, 42, p.1345 (2001).

15. Gómez, C. M. \& Bucnall, C. B. - Polymer, 34, p. 2111 (1993).

16. Girard-Reydet, E.; Riccardi, C. C. \& Sautereau, H. - Macromolecules, 28, p.7608 (1995).

17. Hedreul, C.; Galy, J.; Dupuy, J. \& Delmotte, M. - J. Appl. Polym Sci. 68, p.543 (1998).

18. Turi, E. A. ed. - Thermal Characterization Of Polymeric Materials, Academic Press, New York (1981)

19. Cedeño, A. \& Vazquez-Torres, H. - Polym Int, 54, p.1141 (2005).

20. Kissinger, H. K. - Anal Chem., 29, p.1702 (1957).

21. Ozawa, T. - Bull. Chem. Soc. Jpn., 38, p.1881 (1965).

22. Costa, M. L.; Pardini, L. \& Redenze, M. - Mat. Res., 8, 65 (2005).

23. Schawe, J. E. K. \& Winter, W. - Thermochim Acta, 298, p.9 (1997).

24. Monserrat, S.; Roman, F. \& Colomer, P. - Polymer, 44, p.101 (2003).

25. Horie, K. C.; Hiura, H.; Sawada, M. \& Mita, I. - J. Polym. Sci., Part $A-1,8$, p.1357 (1970).

26. Sourour, S. \& Kamal, M. R. - Thermochim. Acta, 14, p.41 (1976). 
27. Wisanrakitt, G. \& Gillham, J. K. - J. Appl. Polym. Sci., 41, p.2885, (1990).

28. Klute, C. H. \& Vichmann, W. - J. Appl. Polym. Sci. 5, p.86 (1961).

29. Cassetari, M.; Salvetti, G. \& Tombari, E. - J. Polym. Sci., Polym. Phys., 31, p.199 (1993).

30. Swier, S.; Van Assche, G. \& Van Hemelrijck, A. - J. Therm. Anal. 54 p.585 (1998)

31. Monserrat, S. \& Cima, I. - Thermochim. Acta 330, p.189 (1999).

32. Monserrat, S. \& Martín, J. G. - J. Appl. Polym. Sci., 85, p.1263 (2002).

33. Lange, J.; Altmann, N.; Kelly, C. T. \& Halley, P. J. - Polymer, 41, p.5949 (2000).

34. Schawe, J. E. K. - Thermochim. Acta, 361, p.97 (2000).

35. Van Assche, G.; Verdonck, E. \& Van Mele, B. - Polymer, 42, p.2959 (2001).
36. Abuín, S. P.; Pellín, M. P. \& Núñez, L. J. Appl. Polym. Sci. 41, p.2155 (1990).

37. Riccardi, S. S. \& Williams, J. J. - J. Appl. Polym. Sci. 32, p.3445 (1986).

38. Grillet, A. C.; Galy, J.; Pascualt, J. P. \& Bardin, I. - Polymer, 30, p.2094 (1993).

39. Villanueva, M.; Núñez, L. \& Núñez, M. - J. Therm. Anal. and Calorim., 70, p.45 (2002)

40. Gupta, N. \& Varma, I. K. - J. Appl. Polym. Sci. 68, p.1579 (1998).

41. Chern, C. S. \& Poehlein, G. W. - Polym. Eng. Sci. 27, p.788 (1987).

42. Cole, K. C.; Hechler, J. \& Noel, D. - Macromolecules, 24, p.3098 (1991)

Enviado: 04/08/09

Reenviado: 26/03/10

Aceito: $27 / 04 / 10$

DOI: $10.1590 / \mathrm{S} 0104-14282010005000044$ 